\title{
A Clinical Prognostic Scoring System for Neurocysticercosis
}

\author{
Juan Manuel Marquez-Romero ${ }^{1, \odot ~ M a r i ́ a ~ R a q u e l ~ H u e r t a-F r a n c o ~}{ }^{2} \quad$ Elizabeth Soto-Cabrera ${ }^{3}$ \\ Dulce Anabel Espinoza-López ${ }^{3}$ Héctor Orrego ${ }^{3}$ Elizabeth Martínez-Jurado ${ }^{3}$ \\ Fernando Zermeño-Pöhls ${ }^{3}$ Vicente Guerrero-Juárez ${ }^{3}$
}

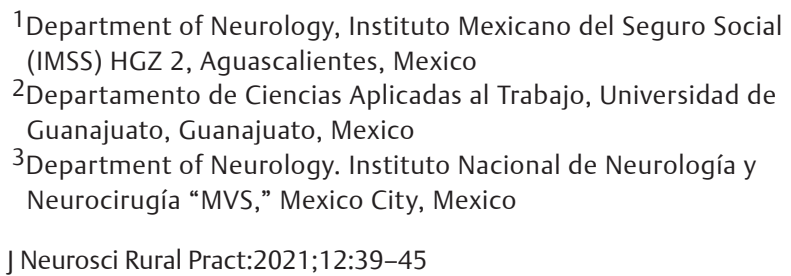

\begin{abstract}
Address for correspondence Juan Manuel Marquez-Romero, MD, MSc, Department of Neurology, Instituto Mexicano del Seguro Social (IMSS) HGZ 2, Av. de Los Conos 102, Ojocaliente, Aguascalientes, 20190, Mexico (e-mail: scint1st@gmail.com).
\end{abstract}

\section{Abstract}

\section{Keywords}

- neurocysticercosis

- prognosis

- risk factors
Objectives In patients with neurocysticercosis (NCC), an accurate risk prediction would allow a better therapeutic approach; however, there are currently no tools that can enhance the accuracy of risk prediction. We designed a prognostic scoring system to be used by neurologists and other physicians managing patients with NCC.

Materials and Methods Using data from clinical records of patients from a third-level national reference center for neurological diseases, we assessed demographic, clinical, and tomographic variables among 293 patients diagnosed with NCC. Multivariable logistic regression analyses were used to develop a clinical prognostic scoring instrument. Patients with NCC were assessed for neurological impairment at 3 months after diagnosis.

Statistical Analysis Score accuracy was assessed by receiver operating characteristic (ROC) curve analysis. The primary outcome was the presence of neurological impairment, resulting in disability according to self-report or caregiver reports; this outcome was assessed during follow-up visits at $3 \pm 1$ months after discharge.

Results The most common symptoms at presentation were headache $(67 \%)$ and seizure $(63 \%)$. A six-item (total score from -4 to +2 ) prognostic instrument was constructed on the basis of the presence of seizures/headache at presentation, a leukocyte count above $12 \times 109 / \mathrm{dL}$, the presence of six to ten parasites, subarachnoid localization, and the use of anthelmintic drugs. Among 113 patients with negative scores, 79.6\% developed neurological deficits. Among patients with scores of 1 to 2 , $64.6 \%$ recovered completely, with an overall accuracy of prediction of $74.7 \%$ and area under the ROC curve $=0.722(95 \% \mathrm{Cl}, 0.664-0.780, p<0.0001)$.

Conclusions The clinical prognostic scoring system for NCC described in this study is a new instrument for use in daily clinical practice. It is simple to administer, and it has a prognostic accuracy of $75 \%$. Its use has the potential to improve the quality of care by guiding appropriate decision-making and early management of patients with NCC.
DOI https://doi.org/ $10.1055 / \mathrm{s}-0040-1718845$ ISSN 0976-3147.

\footnotetext{
(c) 2021. Association for Helping Neurosurgical Sick People.

This is an open access article published by Thieme under the terms of the Creative Commons Attribution-NonDerivative-NonCommercial-License, permitting copying and reproduction so long as the original work is given appropriate credit. Contents may not be used for commercial purposes, or adapted, remixed, transformed or built upon. (https://creativecommons.org/licenses/by-nc-nd/4.0/) Thieme Medical and Scientific Publishers Pvt. Ltd., A-12, 2nd Floor, Sector 2, Noida-201301 UP, India
} 


\section{Introduction}

Neurocysticercosis (NCC) is a highly prevalent parasitosis worldwide $^{1}$ for which there is not a universally accepted treatment regimen. ${ }^{2}$ The lack of consensus about treatment is due to the uncertainty that surrounds its clinical course. The complex interactions between the characteristics of the cysts (size, number and stage of infection) and the host can produce a wide variety of clinical manifestations. These manifestations range from no symptoms to the development of a full-blown immune response against the parasites which usually involves intracranial structures near the cysts. ${ }^{3}$ The development of such an immune response and the degree of the associated inflammation are highly unpredictable.

Current epidemiological information appears to indicate that there is no immune response in most cases; nevertheless, when an immune response does occur, it can lead to a severe, disabling, and potentially lethal condition. ${ }^{4}$ Patients in the extremes of this clinical spectrum are easily identifiable. However, these patients constitute a small portion of all patients with NCC; therefore, more knowledge and experience in treating NCC are necessary for stratifying the risk of neurological impairment for a given patient.

A correct and accurate risk prediction would allow a better therapeutic approach by avoiding the treatment of low-risk patients while maximizing therapeutic efforts in patients at high risk of neurological sequelae.

Data derived from other medical specialties demonstrate that the use of scales to measure or predict risk can improve the accuracy of outcome predictions in a clinical setting, especially for professionals without significant experience. ${ }^{5}$ In addition, prognostic scales can act as standardized measures in patients participating in diagnostic or therapeutic trials.

Despite the significant burden of disease that comes with NCC, risk assessment in patients with the disease continues to be a very difficult task. Therefore, our objective was to develop a simple and reliable prognostic scale that can be of aid in daily clinical practice by studying the risk factor profile of a large sample of patients with NCC who develop neurological impairment.

\section{Materials and Methods}

This study took place in a national reference center for neurological diseases located in Mexico City. We obtained a preliminary list of records from the Department of Epidemiology; the list included all patients presenting for care with NCC according to their discharge diagnosis. Based on that list, the investigators manually reviewed all matching hospital records between January 1, 2006 and November 30, 2009.

All patients included in this study fulfilled the criteria described by Del Brutto et $\mathrm{al}^{6}$ for the diagnosis of NCC. All patients were evaluated by a neurologist who made therapeutic decisions regarding medication use, diagnostic testing, treatment setting (in/out hospital), and need for neurosurgical procedures. All patients underwent at least two noncontrast cranial CT scans, which were the images that were recorded in our database. The attending neurologist also decided the timing and type of antiepileptic drug therapy for each patient regardless of the presence of seizures at presentation.

For each case, we recorded the following variables:

Demographics: age (in years), gender, and place of residence.

Clinical variables: weight in kilograms, height in meters, previous diagnosis of hypertension, diabetes mellitus, smoking, and the reason for presentation to healthcare (by self-report). Regarding treatment, we registered the use of antihelmintic medication, the use of steroids, and the need/ type of surgical intervention (ventriculoperitoneal shunt, cyst extraction).

Laboratory test results: these tests were all performed before the initiation of treatment: initial hemoglobin $(\mathrm{mg} /$ $\mathrm{dL}$ ), white blood cell count, serum eosinophils, and serum glucose $(\mathrm{mg} / \mathrm{dL})$, and in patients who underwent lumbar puncture, cerebrospinal fluid (CSF) glucose, cell count, and protein level.

Cranial CT scan: number of cysts, stage (vesicular, colloid, granular, calcified), cases in whom lesions in more than one stage where present, and location (subarachnoid, intraventricular, parenchymal or a combination of locations). A second investigator was consulted whenever there was doubt about any radiological features of a given case, and disagreements were resolved by consensus.

Clinical outcome: we chose a dichotomous outcome (good/ bad) depending on the presence/absence of neurological impairment assessed during follow-up visits performed at 3 \pm 1 months after discharge. For the purposes of this study, a bad outcome included the persistence of seizures and/or the presence of any other neurological symptoms that precluded the patient from the performance of their premorbid activities of daily living, according to self-report or caregiver report in follow-up visits. Such symptoms included motor deficits, visual field deficits, cognitive impairments, motor coordination deficits, balance impairments, and language disorders.

\section{Statistical Analysis}

We used the Kolmogorov-Smirnov test to examine the distributions of the variables. Continuous variables are described as the mean (standard deviation [SD])/median (range) according to the observed distribution. Categorical variables are described as percentages.

Univariate analyses between groups according to outcome were conducted with the Mann-Whitney U test and the unpaired $t$-test. The Chi-square test was used for categorical comparisons. We created the necessary dummy variables from those variables containing more than one category.

For inclusion in the logistic regression models, significance was considered at $\leq 0.10$; for the purposes of all other statistical analyses, a standard $p \leq 0.05$ was used to indicate significance. Variables that were identified as significant from the univariate analyses were then entered into logistic regression models to identify the independent predictors of either good or bad prognosis. We used stepwise regression with backward 
elimination to construct the models. These models led to a final model with six variables that included one numerical variable; a receiver operating characteristic (ROC) approach was then used to determine an adequate cutoff point and transform the numerical variable into a dichotomous one. Once we obtained the cutoff point, the logistic regression was repeated to obtain a final model that included only dichotomous variables.

Based on the $\beta$ coefficient for each variable included in our final model, a numeric score was assigned to define the scale. Finally, a ROC curve was obtained, and the area under the curve (AUC) was calculated with $95 \%$ confidence intervals.

\section{Results}

We identified and reviewed 322 records from which we eliminated 29 records (9\%); the causes for elimination were an alternative diagnosis ( $n=15,4.7 \%$ ) and a lack of follow-up ( $n=14,4.3 \%)$. Thus, the analysis contains data from 293 patients.

The mean age was $40.4 \pm 14.3$ years, and 141 patients were women $(48.1 \%)$. The most common reasons for presentation for care were headache $(n=195,66.6 \%)$ and seizures $(n=108,63.1 \%)$. The frequencies of diabetes mellitus and hypertension were both $<11 \%$. Blood and serum analysis showed a mean concentration of $14.9 \pm 1.8 \mathrm{mg} / \mathrm{dL}$ for hemoglobin, $9.45 \pm 4.1 \mathrm{~mm}^{3} / \mathrm{dL}$ for white cell count, $0.47 \pm 0.63 \mathrm{~mm}^{3} / \mathrm{dL}$ for eosinophil count, and $112.1 \pm 55.3 \mathrm{mg} / \mathrm{dL}$ for serum glucose.

Regarding the imaging findings, almost $50 \%$ of patients presented a cyst count between two and five. In 20 patients (6.8\%), we observed racemose NCC, and in these cases, it was not possible to accurately count the cysts. We observed symptomatic forms (vesicular and colloid cysts) in $83 \%$ of the cases. The most common localization was subarachnoid in $82 \%$. Eight out of 33 of the subjects with > 10 cysts had parenchymal disease.

Up to $55 \%$ of the patients ( $n=162$ ) received anthelmintics and $82.3 \%$ received steroids $(n=241)$. Among these, 154 patients received a combination of steroids and albendazole, 87 patients received only steroids, and albendazole alone was given to eight patients (2.7\%). No treatment was used in 44 patients (15.0\%). - Table 1 shows all the variables evaluated in the univariate analysis according to clinical prognosis.

A CSF analysis was performed in 99 patients (33.8\%); there were no differences between CSF results according to the outcomes, as shown in - Table 2.

\section{Outcomes}

During the acute phase, 183 patients (62.4\%) developed substantial neurological deficits. This included five deaths (1.7\%), 29 patients who were severely disabled or bedridden (9.9\%), and 149 patients with mild-to-moderate deficits (50.8\%). Nevertheless, these deficits were deemed permanent at the 3-month follow-up in only 151 patients (51.5\%). These were the patients included in the bad outcome category. Epilepsy was the most common diagnosis (29.0\%), followed by chronic headache (20.5\%) and cognitive impairment (8.5\%).

\section{Construction of the Prognostic Scale}

According to the results in the univariate analysis, 13 variables showed an association with the clinical outcome with a significance level of $\leq 0.10$; thus, these variables were entered into the multiple regression procedure. Using backward elimination, those variables with significance levels $\geq 0.05$ were removed from the model. Eventually, only six variables remained that were significantly associated with the clinical outcome and therefore constituted our final model.

The model included seizure and headache at presentation, leukocytosis (> 12x mm $\mathrm{mm}^{3} \mathrm{~L}$ ), subarachnoid localization, a cyst count between 6 and 10 parasites, and the use of anthelmintics ( - Table 3 ).

Based on the $\beta$ coefficient values, we decided to assign the same relative "weight" to each component of the scale. Consequently, a scale can be constructed in which each of the four risk factors for bad outcome corresponds to one negative point or a positive point in the case of the two variables (headache at presentation and use of anthelmintics) that showed association with good outcome ( - Table 4 ).

There were no patients with a score of -4 points on the scale, but among those with a score of -3 points, $90 \%$ developed permanent neurological impairments. Overall, a score below zero points predicted a bad outcome in $79.6 \%$ of the cases. Conversely, $64.6 \%$ of the patients with positive scores recovered completely (-Figs. $\mathbf{1}$ and $\mathbf{2}$ ). Using as threshold values the six scores of the scale found in our patients, a ROC curve was drawn; the AUC was 0.722 (95\% CI: $0.664-0.780$, $p<0.0001$ ).

\section{Discussion}

Based on clinical data easily obtained at bedside or in the consultation room, this study led to the identification of six risk factors strongly associated with the development of permanent neurological impairment in patients with NCC. The obtained model is suitable for use as a score during daily clinical practice.

Concerning the variables that constitute our proposed scoring system, we believe that overall, our results are in accordance with previously known data; for example, Sharma et $\mathrm{al}^{7}$ have previously reported seizures at presentation as one of the major risk factors for developing epilepsy in patients with single granulomas. Nevertheless, these authors also described headache as a factor that was associated with NCC-related epilepsy. The relationship between headache and NCC is very controversial, with some authors considering it to be both a symptom and a sequela of parasitosis. ${ }^{8}$ We tend to believe that although this may indeed be the case, in most patients seeking medical advice on the basis of isolated headache without abnormal findings in the neurological examination, the diagnosis of NCC is usually incidental and unrelated to the symptom, thus explaining why our results predicted a good clinical outcome. Additionally, epidemio$\operatorname{logic}^{4}$ and autopsy studies ${ }^{9}$ have shown that NCC has an asymptomatic course in most patients. With headache being the most common cause of consultation worldwide, it is not surprising in an endemic country such as Mexico to frequently encounter incidental NCC. This is also explained by the increased use of CT 
Table 1 Univariate analysis results according to clinical outcomes

\begin{tabular}{|c|c|c|c|c|c|}
\hline & & All patients & Poor outcome & Good outcome & $p$-Value \\
\hline \multicolumn{6}{|l|}{ Demographic } \\
\hline & Age $^{a}$ & $40(13-85)$ & $40(14-85)$ & & $0.599^{c}$ \\
\hline & Female & $141(48.1)$ & 69 (48.9) & $72(51.1)$ & 0.391 \\
\hline & Outpatient & $98(33.4)$ & $55(36.4)$ & $43(30.3)$ & 0.161 \\
\hline \multicolumn{6}{|l|}{ Clinical } \\
\hline & Seizure & $108(36.9)$ & $82(75.9)$ & $26(24.1)$ & $<0.001$ \\
\hline & Headache & $195(66.6)$ & $74(37.9)$ & $121(62.1)$ & $<0.001$ \\
\hline & Visual impairment & $24(8.2)$ & $13(54.2)$ & $11(45.8)$ & 0.788 \\
\hline & Motor impairment & $23(7.8)$ & $14(60.9)$ & $9(39.1)$ & 0.351 \\
\hline & Sensitive impairment & $7(2.4)$ & $4(57.1)$ & $3(42.9)$ & 0.764 \\
\hline & Transient loss of consciousness & $6(2.0)$ & $4(66.7)$ & $2(33.3)$ & $0.685^{\mathrm{b}}$ \\
\hline & Cerebellar impairment & $3(1.0)$ & 0 & $3(100)$ & $0.113^{b}$ \\
\hline & Neuropsychiatric & $15(5.1)$ & $11(73.3)$ & $4(26.7)$ & 0.083 \\
\hline & Diabetes mellitus & $18(6.1)$ & $9(50.0)$ & $9(50)$ & 0.893 \\
\hline & Hypertension & $31(10.6)$ & $14(45.2)$ & $17(54.8)$ & 0.453 \\
\hline & Smoking & $76(25.9)$ & $46(60.5)$ & $30(39.5)$ & 0.068 \\
\hline \multicolumn{6}{|l|}{ Laboratory } \\
\hline & Hemoglobin (mg/dL) a & $14.9(9.4-19.1)$ & $14.9(3.7-30.8)$ & & $0.135^{c}$ \\
\hline & Leukocytes $(\mathrm{mm} 3 / \mathrm{dL})^{\text {a }}$ & $8.6(3.7-30.8)$ & $8.0(3.7-26.6)$ & & $0.013^{c}$ \\
\hline & Eosinophils (mm3/dL) a & $0.39(0.0-6.0)$ & $0.4(0.0-4.3)$ & & $0.105^{c}$ \\
\hline & Serum glucose $(\mathrm{mg} / \mathrm{dL})^{\text {a }}$ & $97(58-717)$ & $97.5(59-297)$ & & $0.261^{c}$ \\
\hline Lumbar puncture & & $99(33.8)$ & $62(62.6)$ & $37(37.4)$ & 0.007 \\
\hline \multirow[t]{5}{*}{ Cyst number } & Racemose & $20(6.8)$ & $9(45.0)$ & $11(55.0)$ & 0.545 \\
\hline & $>10$ & $33(11.3)$ & $15(45.5)$ & $18(54.5)$ & 0.458 \\
\hline & $6-10$ & $38(13.0)$ & $28(73.7)$ & $10(26.3)$ & 0.003 \\
\hline & $2-5$ & $145(49.5)$ & $79(54.5)$ & $66(45.5)$ & 0.318 \\
\hline & 1 & $95(32.4)$ & $48(50.5)$ & $47(49.5)$ & 0.811 \\
\hline \multirow[t]{4}{*}{ Cyst stage } & Vesicular & $244(83.3)$ & $122(50.0)$ & $112(50.0)$ & 0.240 \\
\hline & Colloid & $27(9.2)$ & $13(48.1)$ & 14 (51.9) & 0.712 \\
\hline & Calcified only & $22(7.5)$ & $16(72.7)$ & $6(27.3)$ & 0.039 \\
\hline & Calcified + any other & $68(23.2)$ & $36(52.9)$ & $32(47.1)$ & 0.791 \\
\hline \multirow[t]{3}{*}{ Cyst location } & Subarachnoid & $239(81.6)$ & $130(54.4)$ & $109(45.6)$ & 0.039 \\
\hline & Intraventricular & $116(39.6)$ & $47(40.5)$ & $69(59.5)$ & 0.002 \\
\hline & Intraparenchymal & $27(9.2)$ & $19(70.4)$ & $8(29.6)$ & 0.040 \\
\hline Anthelmintic use & & 161 (54.9) & $72(44.7)$ & $89(55.3)$ & 0.010 \\
\hline Steroid use & & $239(81.6)$ & $120(50.2)$ & $119(49.8)$ & 0.339 \\
\hline
\end{tabular}

All results to the Chi-squared test unless otherwise specified

avariables are expressed as median (range) and unmarked variables are expressed as $n$ (\%).

bResult to the Fisher exact test

cResult to the Mann-Whitney U test

scans in patients with headaches. Despite the previous data, one must not forget those cases of NCC-related hydrocephalus in whom headache clearly derives from intracranial hypertension caused by an intracranial inflammatory reaction, resulting in a severe clinical form that has been called "malignant NCC" by Estañol et al. ${ }^{10}$ 
Table 2 CSF analysis in 99 patients according to prognosis

\begin{tabular}{|l|l|l|l|}
\hline & Good outcome & Poor outcome & $p$-Value \\
\hline Glucose & $58(11-148)$ & $59(12-379)$ & 0.68 \\
\hline Cellularity & $14(0-234)$ & $5.5(0-253)$ & 0.42 \\
\hline Proteins & $48(6-131)$ & $34(4-183)$ & 0.06 \\
\hline
\end{tabular}

Abbreviation: CSF, cerebrospinal fluid.

Note: All variables median (range). All results to the Mann-Whitney U test

Table 3 Beta coefficients along with the OR and 95\% CI for each variable included in the main and simplified models

\begin{tabular}{|c|c|c|c|}
\hline Variable & $\beta$ Coefficient & $p$-value & OR $(95 \% \mathrm{Cl})$ \\
\hline \multicolumn{4}{|l|}{ Main model ${ }^{\mathrm{a}}$} \\
\hline Seizure at presentation & 1.210 & $<0.001$ & $3.352(1.770-6.348)$ \\
\hline Headache at presentation & -1.060 & 0.002 & $0.347(0.178-.673)$ \\
\hline Leukocytes (mm3/dL) & 0.076 & 0.027 & $0.926(0.866-0.991)$ \\
\hline Presence of $6-10$ cysts & 0.988 & 0.025 & $2.685(1.131-6.373)$ \\
\hline Subarachnoid location of the cysts & 0.784 & 0.034 & $2.191(1.061-4.524)$ \\
\hline Use of anthelmintic medication & -0.637 & 0.023 & $0.529(0.305-0.917)$ \\
\hline Constant & 0.692 & 0.204 & - \\
\hline \multicolumn{4}{|c|}{ Simplified model with all dichotomous variables ${ }^{b}$} \\
\hline Seizure at presentation & 1.162 & $<0.001$ & $3.198(1.683-6.076)$ \\
\hline Headache at presentation & -1.105 & 0.001 & $0.331(0.170-0.647)$ \\
\hline Leukocytes > 12,000 (mm3/dL) & 0.889 & 0.011 & $2.432(1.229-4.814)$ \\
\hline Presence of $6-10$ cysts & 1.097 & 0.015 & $2.995(1.234-7.267)$ \\
\hline Subarachnoid location of the cysts & 0.740 & 0.043 & $2.097(1.022-4.301)$ \\
\hline Use of antihelmintic medication & -0.587 & 0.037 & $0.556(0.320-0.966)$ \\
\hline Constant & -1.585 & 0.042 & - \\
\hline
\end{tabular}

Abbreviations: $\mathrm{Cl}$, confidence interval; OR, odds ratio.

Note: Final result of multiple logistic regression procedures with backward elimination, including the 13 baseline variables listed in $\mathbf{- T a b l e ~} \mathbf{1}$, with $p<0.10$ as initial independent variables.

amain model: overall $R^{2}=0.234 ; p<0.0001$. Patients correctly classified (threshold $=0.5$ ): $75.1 \%$.

bsimplified model: overall $R^{2}=0.238, p<0.0001$. Patients correctly classified (threshold $=0.5$ ): $74.7 \%$.

Table 4 A clinical prognostic score for neurocysticercosis

\begin{tabular}{|l|l|l|}
\hline & $\beta$ & Points \\
\hline $\begin{array}{l}\text { Seizure as initial } \\
\text { manifestation }\end{array}$ & 1.162 & -1 \\
\hline $\begin{array}{l}\text { Headache as initial } \\
\text { manifestation }\end{array}$ & -1.105 & 1 \\
\hline $\begin{array}{l}\text { Leukocytes }>12,000 \\
\text { (mm3/dL) }\end{array}$ & 0.889 & -1 \\
\hline $\begin{array}{l}\text { Presence of } \\
\text { 6-10 cysts }\end{array}$ & 1.097 & -1 \\
\hline $\begin{array}{l}\text { Subarachnoid } \\
\text { location of the cysts }\end{array}$ & 0.740 & -1 \\
\hline $\begin{array}{l}\text { Use of anthelmintic } \\
\text { medication }\end{array}$ & -0.587 & 1 \\
\hline
\end{tabular}

Notably, in the study describing the malignant course of NCC, epilepsy was not considered a bad outcome; nevertheless, modern standards of care deem this approach

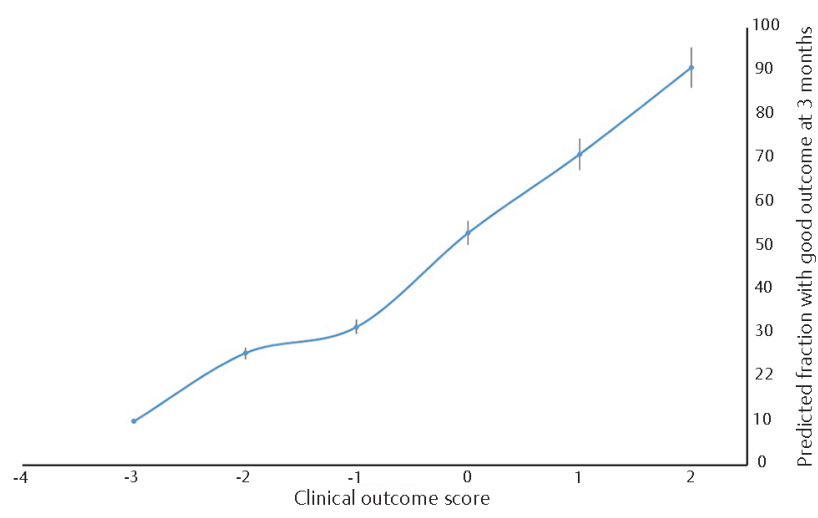

Fig. 1 Predicted fraction of patients with good outcome at 3 months after diagnosis of neurocysticercosis based on the clinical outcome score $(n=293)$.

unacceptable. ${ }^{11}$ Thus, we included epilepsy in our poor outcome definition. Supporting our results, there is data from a randomized controlled trial that identified the 


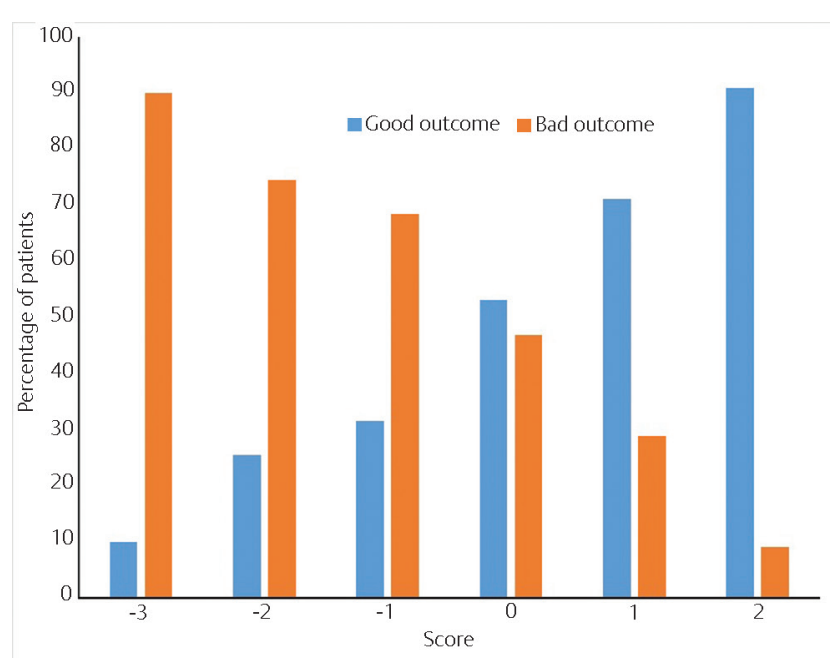

Fig. 2 Totals in the clinical prognostic scoring system by outcome in 293 patients with neurocysticercosis (NCC).

use of anthelmintics as a factor related to the prevention of NCC-related epilepsy. ${ }^{12}$ The incidence of epilepsy has also been shown to be lessened by anthelmintics in patients with single cyst forms ${ }^{13}$ and multiple parenchymal forms ${ }^{14}$ Additionally, current guidelines for the treatment of parenchymal NCC advocate the use of anthelmintics with the purpose of diminishing the number of cysts and the presence of epilepsy. ${ }^{2}$

The cyst location is also critical to the development of neurological sequelae; specifically, parasites with subarachnoid locations can produce more severe symptoms because hyalinized membranes contribute to the formation of an inflammatory exudate that can lead to meningeal thickening and subsequent damage to neural tissue. ${ }^{15}$ This is why subarachnoid NCC, especially that located around the basal cisterns, is regarded as one of the most aggressive forms of the disease. ${ }^{16}$

Regarding the use of steroids, there is a great amount of controversy about their uses and real benefits. Current data and recommendations establish that the role of corticosteroid treatment remains uncertain., ${ }^{2,17}$ Our results appear to support the fact that steroid use plays only a small role, if any, in the clinical outcome of patients with NCC. It has even been suggested that the use of high doses of steroids can lead to the development of NCC forms that are highly resistant to anthelmintics. ${ }^{18}$ We also found an association of leukocytosis with a poor outcome, which has not been previously reported in the literature. This result appears to support the role of systemic inflammation in the development of neurological impairment. Finally, in terms of the variable for the number of cysts, although we found a linear relationship between the number of cysts and clinical impairment, the best predictive value was present among those with a cyst count between six and ten. This finding will require further investigation because many different environmental ${ }^{19}$ and host $^{20}$ factors are associated with cyst burden. Ethnic differences may also play a role because NCC encountered in Asia presents mainly with single cysts, ${ }^{17}$ while in Latin America, most cases carry multiple cysts. ${ }^{21}$ This data may limit the generalizability of our scoring system in Asian endemic countries.

There are very good reasons why our scoring system can be of interest to neurologists. First, its numeric nature provides an objective measure of risk that can serve as a guide to therapeutic decisions; additionally, future researchers can use our system to stratify patients by risk or adjust results from previous trials. Its main strengths are the fact that it only requires simple and routinely obtained data, the ease of calculation, the fact that it is derived from one of the largest sets of data ever published, and that it includes patients within the whole range of NCC manifestations. It also adds to the current evidence on the prognostic role of known variables and adds one previously unknown variable (leukocytosis). However, this scoring system also has some weaknesses that imply caution when interpreting our results and that explain why the accuracy of the score was not higher than $75 \%$. The two main weaknesses that we have identified are the fact that all data was extracted from hospital records (even though each case was evaluated prospectively) and the need to eliminate records with incomplete information or lack of follow-up. Additionally, the selected outcome measure is mainly clinical and does not consider other aspects of the disease, such as the need for in-hospital treatment, requirement of surgical intervention, and the costs associated with all forms of treatment, which are known to be considerable..$^{22}$ Nevertheless, our main goal was to provide neurologists with a clinical tool capable of aiding decision-making during routine clinical duty; hence, our preference for a clinical outcome. These and other aspects indicate the need for prospectively validating these results in a specifically designed cohort, something we intend to do in the future. However, we strongly believe in the usefulness and value of the proposed scoring system and encourage its use among clinicians.

\section{Conclusions}

To conclude, we designed a clinical prognostic scoring system for NCC, which is a new instrument for use in daily clinical practice that has good prognostic accuracy and is simple to administer. Despite its limitations, this scoring system can improve the quality of care of patients with NCC by guiding appropriate early management and eliminating some of the uncertainty that surrounds many aspects of this highly prevalent parasitosis.

\section{Note}

This study was presented at the Neuroinfectious Diseases Section Highlights in the Field Session of the 65th Annual Meeting of the American Academy of Neurology.

\section{Authors' Contributions}

M.R. completed study concept and design, analysis and interpretation of data, writing of the original draft of the manuscript, and study supervision. H.F. took part in study concept and design, analysis and interpretation of data, and critical revision of the manuscript. S.C. contributed in Study concept and design, acquisition of data, and critical revision of the manuscript. D.A.E.L. contributed in 
acquisition of data and critical revision of the manuscript. E.M.J. contributed in the acquisition of data and critical revision of the manuscript. H.O. contributed in of data and critical revision of the manuscript. F.Z.P. contributed in the acquisition of data, critical revision of the manuscript, and study supervision. V.G.J. contributed in the acquisition of data, critical revision of the manuscript, and study supervision.

\section{Ethical Approval}

Under the regulations of the institution where this study took place, institutional review board approval is not mandatory for studies involving only the review of hospital records. No data that might disclose the identity of the subjects under study was recorded. This study was performed conforming to the Declaration of Helsinki.

\section{Conflict of Interest}

None declared.

\section{References}

1 Coyle CM, Mahanty S, Zunt JR, et al. Neurocysticercosis: neglected but not forgotten. PLoSNegl Trop Dis 2012;6(5):e1500

2 Baird RA, Wiebe S, Zunt JR, Halperin JJ, Gronseth G, Roos KL. Evidence-based guideline: treatment of parenchymal neurocysticercosis: report of the Guideline Development Subcommittee of the American Academy of Neurology. Neurology 2013;80(15):1424-1429

3 Mahanty S, Garcia HH; Cysticercosis Working Group in Perú. Cysticercosis and neurocysticercosis as pathogens affecting the nervous system. Prog Neurobiol 2010;91(2):172-184

4 Garcia HH, Del Brutto OH; Cysticercosis Working Group in Peru. Neurocysticercosis: updated concepts about an old disease. Lancet Neurol 2005;4(10):653-661

5 McClish DK, Powell SH. How well can physicians estimate mortality in a medical intensive care unit. ? Med Decis Making 1989;9(2):125-132

6 Del Brutto OH, Rajshekhar V, White AC Jr, et al. Proposed diagnostic criteria for neurocysticercosis. Neurology 2001; 57(2):177-183

7 Sharma P, Garg RK, Verma R, Singh MK, Shukla R. Risk of seizure recurrence in patients of new-onset partial seizure having a solitary cysticercus granuloma of brain or normal neuroimaging. J Neurol Sci 2011;301(1-2):21-26

8 Del Brutto OH. Neurocysticercosis: new thoughts on controversial issues. Curr Opin Neurol 2013;26(3):289-294
9 de Almeida SM, Torres LF. Neurocysticercosis-retrospective study of autopsy reports, a 17-year experience. J Community Health 2011;36(5):698-702

10 Estañol B, Corona T, Abad P. A prognostic classification of cerebral cysticercosis: therapeutic implications. J Neurol Neurosurg Psychiatry 1986;49(10):1131-1134

11 Garcia HH. Interview-Hector H. Garcia. Interviewed by Christo Hall. Pathog Glob Health 2012;106(5):254-255

12 Garcia HH, Pretell EJ, Gilman RH, et al; Cysticercosis Working Group in Peru. A trial of antiparasitic treatment to reduce the rate of seizures due to cerebral cysticercosis. $\mathrm{N}$ Engl J Med 2004;350(3):249-258

13 Goel D, Mittal M, Bansal KK, Singhal A. Natural history of solitary cerebral cysticercosis cases after albendazole therapy: a longitudinal follow-up study from India. Acta Neurol Scand 2010;121(3):204-208

14 Kelvin EA, Carpio A, Bagiella E, et al; Ecuadorian Neurocysticercosis Group. Seizure in people with newly diagnosed active or transitional neurocysticercosis. Seizure 2011; 20(2):119-125

15 Del Brutto OH. Neurocysticercosis: a review. ScientificWorldJournal 2012;2012:159821

16 Fleury A, Carrillo-Mezo R, Flisser A, Sciutto E, Corona T. Subarachnoid basal neurocysticercosis: a focus on the most severe form of the disease. Expert Rev Anti Infect Ther 2011;9(1):123-133

17 Otte WM, Singla M, Sander JW, Singh G. Drug therapy for solitary cysticercus granuloma: a systematic review and meta-analysis. Neurology 2013;80(2):152-162

18 Cárdenas G, Carrillo-Mezo R, Jung H, Sciutto E, Hernandez JL, Fleury A. Subarachnoidal Neurocysticercosis non-responsive to cysticidal drugs: a case series. BMC Neurol 2010;10:16

19 Kelvin EA, Yung J, Fong MW, et al. The association of living conditions and lifestyle factors with burden of cysts among neurocysticercosis patients in Ecuador. Trans R Soc Trop Med Hyg 2012;106(12):763-769

20 Kelvin EA, Carpio A, Bagiella E, et al. Ecuadorian Neurocysticercosis Group. The association of host age and gender with inflammation around neurocysticercosis cysts. Ann Trop Med Parasitol 2009;103(6):487-499

21 Fleury A, Moreno García J, Valdez Aguerrebere P, et al. Neurocysticercosis, a persisting health problem in Mexico. PLoS Negl Trop Dis 2010;4(8):e805

22 Croker C, Reporter R, Mascola L. Use of statewide hospital discharge data to evaluate the economic burden of neurocysticercosis in Los Angeles County (1991-2008) Am J Trop Med Hyg 2010;83(1):106-110 